\section{Chaperone required?}

Like any parent keeping an eye on their child, molecular chaperones are recruited to stop anything untoward happening to a protein. By binding the protein, they keep it folded and protected from degradation or unsuitable interactions. They are not required for protein function per se but, without their protection, the proteins are certain to get into trouble. It is equally important that chaperones know when to let go and, for most chaperones identified so far, this has been shown to be regulated by an ATPase cycle. But now, reporting in Cell, Tsai and colleagues have identified a chaperone protein disulphide isomerase (PDI) - that is unique in many ways. Not only does it promote unfolding of its target, the cholera toxin Al chain, but it is also regulated by a distinct mechanism - a redox cycle.

The cholera holotoxin, produced by Vibrio cholerae, consists of $\mathrm{B}$-chain subunits bound to an $A 1$ and $A 2$ chain, which in turn are joined by disulphide bonds. Upon uptake by a host cell, the holotoxin undergoes a plethora of modifications, culminating in the release of the active toxin - the Al chain - into the cytosol, where it ultimately causes opening of chloride channels and massive secretion of water and chloride. While much is known about how $\mathrm{A} 1$ functions in the cytosol, less is known about how it is released from the rest of the holotoxin and unfolded in the endoplasmic reticulum (ER).

To decipher how this occurs, the authors developed an in vitro assay to look for triggers

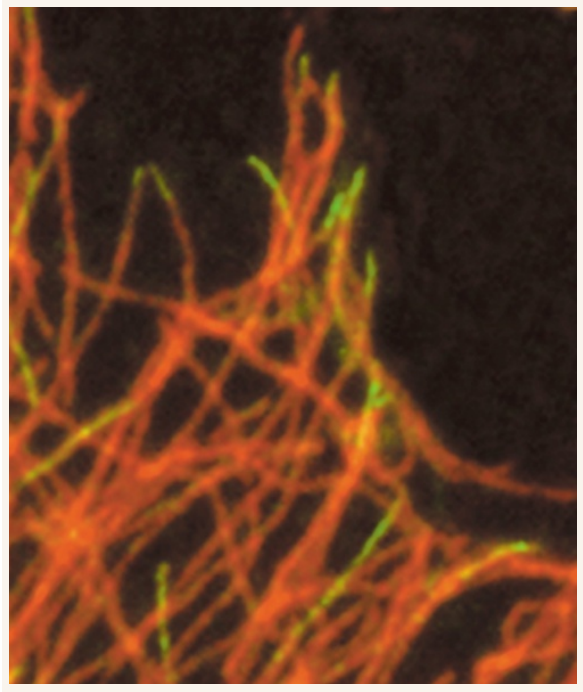

GFP-CLASP1 at the ends of microtubules (red). Courtesy of Ann Akhmanova, Erasmus University, Rotterdam, The Netherlands. of A1 release. They bound the purified holotoxin to beads and then looked at the effects of adding different extracts. Whereas addition of bovine serum albumin did nothing, addition of an ER lumenal extract caused release of a significant fraction of the A1 chain. The ER extract also rendered the A chain protease sensitive. To isolate the unfolding activity within this extract, they eluted the proteins on a salt gradient, tested each fraction again for activity and then analysed the fraction showing activity by mass spectrometry. It turned out to be PDI, which belongs to a family of oxidoreductases. To confirm that they had purified the right thing, they showed that immunodepleting PDI blocked the release of A1.

As PDI is an oxidoreductase, Tsai and coworkers wondered whether its unfolding activity is dependent on the redox potential. Unfolding activity, they found, was dependent on reducing conditions. Similarly, they showed that binding of PDI to the A subunit occurs only under reducing conditions and that this binding is reversed when conditions become oxidizing.

Previous studies indicate that cleavage of the A subunit into the $\mathrm{A} 1$ and $\mathrm{A} 2$ chains is a prerequisite for toxin activation. Must this also occur for PDI-induced unfolding? To test this, the authors mutated the site in the A chain - the Cys187-Cys199 loop - that is cleaved to produce the A1 and A2 chains. And they found that this disrupted binding and unfolding, indicat-

\section{CYTOSKELETON}

\section{Clasping on to the end}

Whereas the minus ends of microtubules are anchored to microtubule-organizing centres, their plus ends are free and can either grow or shrink rapidly. Several proteins, including CLIP170 and its neuronal homologue CLIP-115, are known to bind the plus ends of growing microtubules. Akhmanova and colleagues now report in Cell that they have found two more proteins that seem to share this characteristic.

The authors found CLASP 1 and CLASP 2 in a yeast two-hybrid screen for proteins interacting with a conserved region of CLIP-115, and confirmed that CLASPs bind directly to CLIPs. The association of CLASPs with the tips of microtubules is direct and does not depend on CLIPs. In

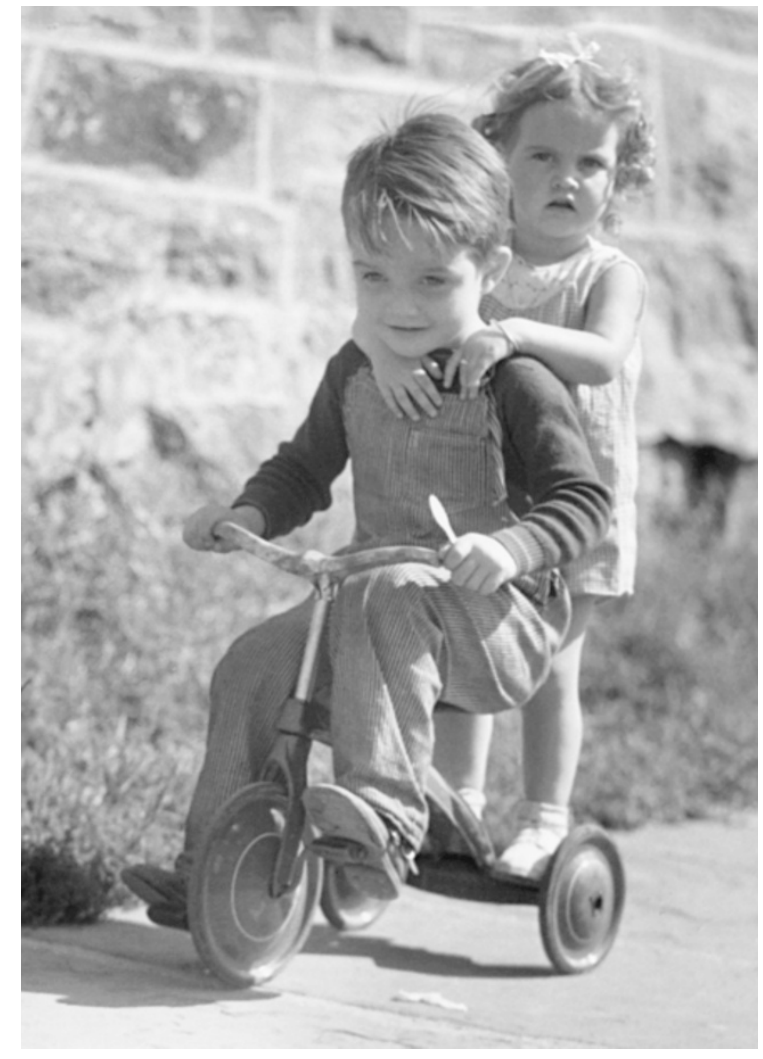

ing that cleavage is indeed a prerequisite for recognition by PDI.

The authors propose "that PDI is a chaperone that changes its affinity for substrates during cycles of oxidation and reduction of its disulfide bridges". This is similar to the ATP binding cycle of conventional chaperones, and reveals a common theme - both cycles are driven by energy. The authors speculate that this non-equilibrium state might allow cycles of binding and release as well as regulation of these processes. How common this type of chaperone is remains to be seen.

Q) References and links

Alison Schuldt Original Research PAPER Tsai, B. et al. Protein disulfide isomerase acts as a redox-dependent chaperone to unfold cholera toxin. Cell 104, 937-948 (2001) addition, CLASPs seem to have microtubule-stabilizing properties.

Unlike CLIPs, which label growing microtubule ends throughout the cell, CLASPs specifically localize to microtubule ends at the leading edge of motile cells. Interaction of CLASPs with microtubules is influenced by phosphorylation, and the authors provide evidence that inhibition of glycogen synthase kinase-3b by phosphatidylinositol 3-kinase (PI3K) might regulate this association. These findings provide a possible mechanism for the previously described action of PI3K in the response of fibroblasts to positional cues.

(2) References and links Raluca Gagescu ORIGINAL RESEARCH PAPER Akhmanova, A. et al. CLASPs are CLIP-115 and -170 associating proteins involved in the regional regulation of microtubule dynamics in motile fibroblasts. Cell 104, 923-935 (2001) 